\title{
Challenges to the Violation-of-Expectation Paradigm: Throwing the Conceptual Baby Out With the Perceptual Processing Bathwater?
}

\author{
Yuko Munakata \\ Department of Psychology \\ University of Denver
}

Assessing infant knowledge is notoriously difficult. The ambiguities in infants' limited repertoire of behaviors have sparked some of the fiercest debates in cognitive development (e.g., Diamond, 1991; Haith, 1998; Meltzoff \& Moore, 1977; Piaget, 1954; Rovee-Collier, 1997; Spelke, 1998; Thelen \& Smith, 1994; Wynn, 1992). On the one hand, researchers who rely on relatively simple measures to assess infant knowledge are accused of "rich interpretation" (Haith \& Benson, 1997), attributing conceptual understanding to infants when more basic processes could subserve their behavior. On the other hand, researchers who discount findings from simpler measures and rely on relatively strict measures to assess infant knowledge are accused of proposing "deflationary accounts" (Scholl \& Leslie, 1999), stretching nonconceptual accounts beyond their limits to avoid attributing knowledge to infants. Such challenges have provoked stimulating debates about what infants know, together with active attempts to resolve these debates empirically. This work holds the promise of significantly advancing our understanding of the origins and nature of our knowledge.

The preceding collection of articles contributes to this important endeavor with a coherent set of studies using the violation-of-expectation paradigm. Each article presents a variant of the well-known drawbridge paradigm (Baillargeon, 1987, 1993; Baillargeon, Spelke, \& Wasserman, 1985) and argues that the resulting data support an alternative, nonconceptual view of infant looking times in these studies.

Requests for reprints should be sent to Yuko Munakata, Department of Psychology, University of Colorado, 2155 South Race Street, Denver, CO 80208. E-mail: munakata@du.edu 
Specifically, rather than infants looking longer at events based on their possibility-impossibility, which would suggest a conceptual understanding of the permanence and solidity of objects, infants look longer at events based on their familiarity-novelty, suggesting the operation of simpler, perceptual processes. This set of studies forms a compelling package that highlights the importance of considering a variety of factors that influence infant behavior. Any complete theory of infant behavior in violation-of- expectation studies will need to account for infants' preferences for familiarity-novelty.

As compelling as these studies are, however, one must be careful not to throw out the baby with the bath water, that is, the conceptual baby with the perceptual processing bath water. Several aspects of this work limit the conclusions that can be drawn from it at this time. As elaborated in the following, these limitations stem from (a) failures to address a critical control condition; (b) failures to replicate the original findings, making it difficult to isolate the critical differences between studies; and (c) mischaracterizations of conceptual accounts as positing that only conceptual factors matter. As a result of these limitations, the preceding collection of articles may best be viewed as demonstrating that perceptual processing factors influence infants' looking times in violation-of-expectation studies, nonetheless allowing for the possibility that infants are additionally sensitive to the impossibility of events.

\section{FAILURES TO ADDRESS A CRITICAL CONTROL CONDITION}

A primary limitation of perceptual processing accounts (as proposed in the articles in this collection, as well as elsewhere; e.g., Rivera, Wakely, \& Langer, 1999) is their failure to address a control condition designed specifically to counter such accounts. In this control condition (Baillargeon et al., 1985), infants were habituated to a full $180^{\circ}$ rotation of the drawbridge as in the experimental condition (Baillargeon, 1987; Baillargeon et al., 1985). At test, a block was placed to the left of the drawbridge. The drawbridge then repeatedly rotated fully $\left(180^{\circ}\right)$ or partially $\left(112^{\circ}\right)$, with the block visible to the side of the drawbridge throughout the events. Both the full- and partial-rotation events were possible, because the block was out of the path of the drawbridge. Under these control conditions, infants looked equally at the full- and partial-rotation events. These findings are critical because the elements of the experimental and control test events were identical, but only the full-rotation event in the experimental condition was impossible. Thus, infants' longer looking to the impossible, full rotation could not be due to a simple preference for a full rotation, nor to a preference for familiar events.

Although conceptual accounts explained these findings in terms of the possibility of the events, it appears that perceptual processing accounts cannot readily ex- 
plain the findings of the critical control condition. Instead, perceptual processing accounts appear to mistakenly predict that the control condition should yield the same results as the experimental condition. For example, if infants simply prefer to look at full drawbridge rotations (Rivera et al., 1999), they should have looked longer at the full rotation in both the experimental and control conditions. Similarly, if infants simply respond to perceptual changes from habituation to test (Bogartz et al., this issue; Cohen \& Cashon, this issue; Schilling, this issue), longer looking to the full rotation in the experimental condition should have been paralleled with longer looking to the full rotation in the control condition, because these events were equivalent with respect to the novelty of the block and the familiarity of the rotation. Instead, infants looked longer at the full rotation only in the experimental condition - a result perfectly consistent with the conceptual interpretation but inconsistent with perceptual processing accounts.

This control condition appears to have been overlooked by alternative accounts because they have focused on the possible and impossible events of the experimental condition. Although such alternative accounts have correctly emphasized that the possibility of these events is confounded with other factors (familiarity, degree of screen rotation, etc.), they have failed to explain the findings from the control condition designed to address exactly these kinds of factors. Until the findings from the control condition can be explained within perceptual processing accounts, the conceptual account appears to provide a better explanation for the original set of findings than do the proposed alternatives.

\section{FAILURES TO REPLICATE THE ORIGINAL FINDINGS}

Of course, the perceptual processing accounts propose to go beyond simply accounting for the original findings - they are also aimed at providing a coherent framework for understanding new, interesting results such as those reported in the preceding set of articles. Specifically, infant preferences for familiarity-novelty appear to explain infant looking in violation-of-expectation studies employing an Event Set $\times$ Event Set design (Bogartz, Shinskey, \& Speaker, 1997) in which multiple events serve as habituation and test events (Bogartz et al., this issue; Cashon \& Cohen, this issue) and in more traditional designs with varying numbers of familiarization trials (Schilling, this issue). Such findings bolster the claim that infant looking reflects the familiarity of events coupled with infant preferences for familiarity-novelty.

However, researchers have yet to provide the most compelling kind of support for this claim. Such support would consist of (a) a replication of the original methods and findings for validation purposes, (b) additional data collected with systematic manipulation of aspects of the original methods, and (c) analyses indicating that perceptual processing factors best predict looking times across (a) and (b). The 
current support for perceptual processing accounts falls short of this compelling package by skipping the first step. As a result, these data show only that with different methods (and in most cases, failures to replicate), perceptual factors are the best predictor of infant looking. ${ }^{1}$ These methodological differences make it difficult to determine the generality of the findings. Are perceptual processing factors the best predictor of infant looking across all violation-of-expectation studies, or only when methods have been changed such that infants fail to look longer at impossible events, failing to replicate the original findings?

For example, in the original studies, the block was a 3-D, solid object that infants were able to manipulate prior to the experiment; in contrast, the block in these articles was either essentially 2-D (a piece of $1 \mathrm{~cm}$ thick foam core; Bogartz et al., this issue; Schilling, this issue) or computer-generated (Cashon \& Cohen, this issue). Impossible events violating the permanence and solidity of a 2-D or computer block seem unlikely to be as compelling as those with a 3-D block that infants have manipulated. Thus, infants in these studies may have been sensitive to object permanence and solidity but did not find the 2-D and computer events compellingly impossible, such that their looking times instead reflected simple familiarity. ${ }^{2}$

The studies reported in this collection of articles also differ from the original studies in the procedure at the start of each trial. In the original studies, infants first had to look at the block for $2 \mathrm{sec}$ before the event started. None of these studies appeared to apply this criterion. This difference may be negligible (infants did distinguish events with and without the block in some of the studies, indicating that they must have noticed the block), or it may be critical to recognizing the impossibility of events (e.g., infants may use the time before the drawbridge starts moving to form predictions that allow them to recognize the impossibility of events; Munakata, in press).

These kinds of methodological differences, combined with the failures to replicate the original findings, make it difficult to understand the role of perceptual processing factors in infant looking times. Do infants simply fall back on such factors when impossible events do not really look impossible, when events begin without

\footnotetext{
${ }^{1}$ Although one condition in one study (Schilling, this issue) appeared to replicate the original findings, it is unclear whether these results constitute a true replication, given numerous differences between these methods and the original. Therefore, infants may have looked longer at impossible events in this study due to familiarity (as proposed by Schilling), whereas they may have looked longer at impossible events in the original, methodologically different, studies due to conceptual understanding.

${ }^{2}$ Cashon and Cohen (this issue) make the important point that infants have shown sensitivity to other factors (e.g., causality and object unity) in studies with computer-animated stimuli, suggesting that computer-animated stimuli can serve as valid test materials. However, some domains may lend themselves more readily than others to testing with computer-animated stimuli. It seems possible that adult judgments would parallel infants' apparent sensitivity, with adults similarly interpreting computer-generated events in terms of causality and object unity, but not finding the impossible computer-animated drawbridge event particularly compelling.
} 
infants having a chance to encode the display, and so on? Or, are perceptual processing factors the best predictor of infant looking, even in cases that have been interpreted in terms of infants' conceptual understanding? To argue the latter, researchers need to more closely replicate the original methods and findings to demonstrate the generality of their claims.

\section{MISCHARACTERIZATIONS OF CONCEPTUAL ACCOUNTS}

Finally, conceptual accounts are often mischaracterized as positing that only conceptual factors affect infant looking, such that if researchers demonstrate the importance of other factors such as familiarity, that poses a challenge to conceptual accounts. However, proponents of conceptual accounts would not deny the importance of factors such as familiarity. Familiarity is in fact a consideration in many violation-of-expectation studies-infants are often habituated to the motion of objects (e.g., the rotating motion of the drawbridge) specifically so that this motion becomes familiar, allowing infants' looking times at test to better reflect their sensitivity to the possibility of events. Thus, demonstrating the importance of familiarity need not rule out potential contributions from other factors, such as conceptual understanding.

\section{CONCLUSIONS}

In sum, a more compelling case for the proposed alternative perceptual processing account will require (a) the consideration of the broad spectrum of behavioral data, including the critical control condition that seems to challenge alternative accounts; (b) the replication of the original methods and results to demonstrate the generality of the claims; and (c) not setting up conceptual and perceptual accounts as mutually exclusive-evidence for one should not be interpreted as ruling out the other.

As it stands, the preceding collection of articles provides a stimulating package that raises interesting alternative interpretations of infant looking in violation-of-expectation studies and highlights the importance of considering multiple factors that influence behavior. This work emphasizes the need for theoretical approaches that can capture the complexity of interactions among many such factors in development (e.g., the neural network modeling framework, Elman et al., 1996; Munakata \& Stedron, in press; and the dynamic systems framework, Thelen \& Smith, 1994). However the debates about violation-of-expectation studies are ultimately resolved, the preceding articles provide an important contribution to our understanding of the factors influencing infant 
looking, and in turn, should help to address some of the most fiercely debated issues in cognitive development.

\section{ACKNOWLEDGMENTS}

Preparation of this commentary was supported by grants from the National Institute of Child Health and Development (1R29 HD37163-01) and the National Science Foundation (IBN- 9873492).

I thank Randy O'Reilly, Jennifer Stedron, Marshall Haith, and Janette Benson for useful feedback.

\section{REFERENCES}

Baillargeon, R. (1987). Object permanence in 3.5- and 4.5-month-old infants. Developmental Psychology, 23, 655-664.

Baillargeon, R. (1993). The object concept revisited: New directions in the investigation of infants' physical knowledge. In C. Granrud (Ed.), Visual perception and cognition in infancy: Carnegie Mellon Symposia on Cognition (pp. 265-315). Hillsdale, NJ: Lawrence Erlbaum Associates, Inc.

Baillargeon, R., Spelke, E., \& Wasserman, S. (1985). Object permanence in five-month-old infants. Cognition, 20, 191-208.

Bogartz, R. S., Shinskey, J. L., \& Speaker, C. (1997). Interpreting infant looking: The Event Set $\times$ Event Set design. Developmental Psychology, 33, 408-422.

Diamond, A. (1991). Neuropsychological insights into the meaning of object concept development. In S. Carey \& R. Gelman (Eds.), The epigenesis of mind (pp. 67-110). Hillsdale, NJ: Lawrence Erlbaum Associates, Inc.

Elman, J., Bates, E., Karmiloff-Smith, A., Johnson, M., Parisi, D., \& Plunkett, K. (1996). Rethinking innateness: A connectionist perspective on development. Cambridge, MA: MIT Press.

Haith, M. M. (1998). Who put the cog in infant cognition? Is rich interpretation too costly? Infant Behavior and Development, 21, 167-179.

Haith, M. M., \& Benson, J. (1997). Infant cognition. In W. Damon (Series Ed.) \& R. Siegler \& D. Kuhn (Vol. Eds.), Handbook of child psychology: Vol. 2. Cognition, perception, and language (5th ed., pp. 199-254). New York: Wiley.

Meltzoff, A., \& Moore, M. (1977). Imitation of facial and manual gestures by human neonates. Science, 198, 75-78.

Munakata, Y. (in press). Task-dependency in infant behavior: Toward an understanding of the processes underlying cognitive development. In F. Lacerda, C. v. Hofsten, \& M. Heimann (Eds.), Emerging cognitive abilities in early infancy (pp. 29-52). Mahwah, NJ: Lawrence Erlbaum Associates, Inc.

Munakata, Y., \& Stedron, J. M. (in press). Neural network models of cognitive development. In C. A. Nelson \& M. Luciana (Eds.), Handbook of developmental cognitive neuroscience. Cambridge, MA: MIT Press.

Piaget, J. (1954). The construction of reality in the child. New York: Basic Books.

Rivera, S. M., Wakeley, A., \& Langer, J. (1999). The drawbridge phenomenon: Representational reasoning or perceptual preferences? Developmental Psychology, 35, 427-435.

Rovee-Collier, C. (1997). Dissociations in infant memory: Rethinking the development of implicit and explicit memory. Psychological Review, 104, 467-498. 
Scholl, B., \& Leslie, A. (1999). Explaining the infant's object concept: Beyond the perception/cognition dichotomy. In E. Lepore \& Z. Pylyshyn (Eds.), What is cognitive science? (pp. 26-73). Oxford, England: Blackwell.

Spelke, E. (1998). Nativism, empiricism, and the origins of knowledge. Infant Behavior and Development, 21, 181-200.

Thelen, E., \& Smith, L. B. (1994). A dynamic systems approach to the development of cognition and action. Cambridge, MA: MIT Press.

Wynn, K. (1992). Addition and subtraction by human infants. Nature, 358, 749-750. 